\title{
Parabolic Equation Accumulated Split Error and Its Correction Method for Tropospheric Radio Propagation
}

\author{
Peipei Wei $\mathbb{D}^{1},{ }^{1}$ Xiaoyan Du $\mathbb{D},{ }^{2}$ Xinwei Hu $\mathbb{D},{ }^{1}$ and Changyin Jiang $\mathbb{D}^{3}$ \\ ${ }^{1}$ School of Zhengzhou Information Science and Technology Institute, Zhengzhou, China \\ ${ }^{2}$ University of Zhengzhou, Zhengzhou, China \\ ${ }^{3}$ China Research Institute of Radio Wave Propagation, Xinxiang, China
}

Correspondence should be addressed to Xiaoyan Du; 15713830138@163.com

Received 22 October 2017; Accepted 21 January 2018; Published 2 April 2018

Academic Editor: Sotirios K. Goudos

Copyright (c) 2018 Peipei Wei et al. This is an open access article distributed under the Creative Commons Attribution License, which permits unrestricted use, distribution, and reproduction in any medium, provided the original work is properly cited.

An increasingly popular approach of modeling electromagnetic wave propagation in the troposphere is to use the slip-step Fourier transform (SSFT) numerical algorithm to solve a parabolic equation (PE). However, available SSFT does not provide perfect solutions by introducing accumulated split error (ASE) for its step-by-step iteration process with a fixed propagation range step. The common analysis on one step split error is only a simple discussion on the split error of PE. Therefore, the main motivation of this study is to provide improvement on the accuracy of the PE by proposing an error correction method for the first time in the literature for PE. This method is on the basis of defining and deriving the accumulated split error. Its performance is then demonstrated with measured radar sea clutter data from Huanghai Sea, China.

\section{Introduction}

Most radio systems have to operate in the lower tropospheric atmosphere. Atmosphere absorbing, scattering, reflection, and refraction could affect radio propagation characteristics. In particular, the occurrence of tropospheric duct can make radio wave beyond-line-of-sight propagation [1], extending communication range and detection distance of radar system. This phenomenon also may cause radio holes and introduce new interfaces, which fundamentally can change the performance of a system that is designed to operate under standard atmospheric conditions [1]. This nonstandard atmosphere has a higher demand on effectively describing propagation characteristics.

Over time, radio propagation modeling methods in tropospheric environment mainly focus on ray tracing (RT) $[2,3]$ and parabolic equation $(\mathrm{PE})[4,5]$ methods. RT generally neglects the influence of frequency and fails to describe the distribution of field strength at arbitrary location because of restricting to ray numbers. PE is a full-forward-wave approximation calculation method which is derived from wave equation using paraxial approximation [5]. It can deal with electromagnetic propagation problems in arbitrary irregular terrain and inhomogeneous atmosphere with the help of computer numeric calculation. Therefore, PE has been the most popular approach for tropospheric electromagnetic propagation [1-10]. According to propagation angle applied to the Helmholtz wave equation to obtain PE, $\mathrm{PE}$ can be divided into wide-angle PE (WPE) and narrowangel PE (or standard PE (SPE)).

At present, there are mainly three algorithms to solve PE: split-step Fourier transform (SSFT) [1], finite difference schemes [11], and finite element method [12], where SSFT has the advantage of higher numerical stability and thus is widely used to solve PE for tropospheric propagation problems. However, PE has approximation error due to paraxial approximation and split error as a result of exponent operator split using SSFT, where approximation error is its inherent drawback. For the past work on the error analysis of PE, approximation error is lower for SPE, and it can be ignored [13]. The expression of one-step split error of SPE is described in [14]. The two kinds of errors for WPE and 
SPE are discussed with simple calculation in [4]. Some advices are proposed to decrease the split error by studying its relationship with range step in [15]. However, an analytical description of the accumulation of split error (called accumulated split error (ASE)) over many range steps has yet to be successfully developed. In summary, these studies are inefficient to degrade split error. For this problem, we conduct the research on ASE and provide its correction method in this paper. In our work, the accumulated split error of SPE is derived for the first time in the literature on account of the definition of split error [4] and the iteration idea of SSFT. The derivation result shows that the ASE is determined by the atmosphere refractivity, the antenna frequency, the propagation elevation angle, the range step, and the field strength with split error. We simulate the sensitivity of ASE resulting from these factors using two statistical methods in a standard and nonstandard atmosphere. With the research conclusions, the ASE can be degraded by taking small range step in a standard atmosphere. However, this solution does not work well for nonstandard atmosphere on some conditions. For solving this problem, an ASE correction method is provided. This method is involved in complex calculation, including higher order accumulation, multiplication, and power operation. In addition, some supplements are provided for this method to analyze the complexity and efficiency. Finally, this method is verified by measured radar sea clutter from Huanghai Sea, China.

The main contributions of this paper are fourfold. Firstly, we derive the accumulated split error of SPE for the first time in the literature. Secondly, the sensitivity analysis of ASE in a standard and nonstandard atmosphere is discussed using two statistical methods. Thirdly, we develop an ASE correction method to improve the accuracy of PE. Lastly, radar sea clutter power calculated by our proposed approach and past $\mathrm{PE}$ method is compared with the measured data from Huanghai Sea China, respectively.

The remainder of the paper is organized as follows. Section 2 presents the deviation of the ASE and its iteration model. Section 3 provides simulation results of sensitivity analysis. In Section 4, the ASE correction method is introduced. In addition, experiment research is provided. Lastly, the conclusions are presented in Section 5.

\section{Parabolic Equation Accumulated Split Error}

This section presents the derivation of the ASE on the basis of the one split error of PE.

2.1. Parabolic Approximation. In the rectangular coordinate system shown in Figure 1, the SPE for the field $u(x, z)$ is expressed as [5].

$$
\frac{\partial^{2} u(x, z)}{\partial z^{2}}+2 i k \frac{\partial u(x, z)}{\partial x}+k^{2}\left(n^{2}-1+\frac{2 z}{a}\right) u(x, z)=0,
$$

where $k$ is the wave number and $k=2 \pi / \lambda$ ( $\lambda$ is the wavelength.), $a$ is the Earth's radius, and $n$ is the refractive index. The term $2 z / a$ accounts for the spherical shape of

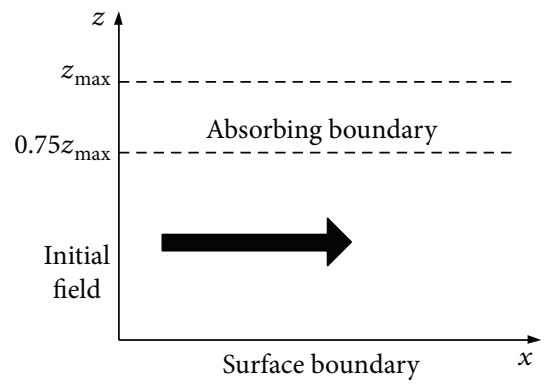

Figure 1: The calculation domain of PE.

the Earth. When it is omitted, (1) describes propagation over a flat Earth.

Only considering the forward wave propagation, (2) is obtained after simple transform of (1)

$$
\frac{\partial u(x, z)}{\partial x}=(A+B) u(x, z)
$$

where the expressions of two operator terms $A$ and $B$ are

$$
\begin{aligned}
& A=\frac{i k}{2} w, \\
& B=\frac{i}{2 k} \frac{\partial^{2}}{\partial z^{2}},
\end{aligned}
$$

with $w=n^{2}(x, z)-1+(2 z / a)$. The horizontally inhomogeneous refractivity index $n$ is considered here, shown as $n(x, z)$.

Integrate over $x$ for (2), then the solution is given as

$$
u(x+\Delta x, z)=\exp [\Delta x(A+B)] u(x, z)
$$

where $\Delta x$ is the range step.

In order to solve this formula, error is incurred by splitting the exponential with the hypothesis that $n$ is a constant. The simplest split is given by [4].

$$
\exp [\Delta x(A+B)] \approx \exp (\Delta x A) \exp (\Delta x B) .
$$

Then the expression that can be solved is provided by

$$
u(x+\Delta x, z)=\exp (\Delta x A) \exp (\Delta x B) u(x, z) .
$$

Using (6) and other boundary conditions shown in Figure 1, the field can be described in the propagation domain [5]. It is the commonly used method.

The term $w$ is varied with height in the realistic environment, then

$$
\frac{\partial^{2}[w u]}{\partial z^{2}} \neq(w) \frac{\partial^{2} u}{\partial z^{2}}
$$

Therefore, the two terms splitting in the exponent is not exact in nonhomogeneous medium. The split error $E_{\text {error }}$ is provided as

$$
E_{\text {error }}=e^{(B+A) \Delta x} u(x, z)-e^{A \Delta x} e^{B \Delta x} u(x, z) .
$$

This formula is the one-step split error at the range $x$ assuming that the field $u(x, z)$ is the same for the right- 
hand side of (8). However, the field strength values of $u(x, z)$ are different for the right-hand sides of (4) and (6), except for the initial field $u(0, z)$ at the range $x=0$. Therefore, (8) is not applied for correcting the ASE between (4) and (6) over many range steps.

2.2. Accumulated Split Error Modeling. We assume that there is a solution meeting (4), then

$$
u_{j}=\exp [\Delta x(A+B)] u_{j-1}=F_{1}\left\langle u_{j-1}\right\rangle,
$$

where $F_{1}=\exp [\Delta x(A+B)]$, and $u_{j}$ is the field at the $j$ th $\left(0 \leq j \leq N_{x}-1, N_{x}\right.$ is the horizontal grid points) range step, which gives the result using PE without ASE.

Let $u_{j}^{\prime}$ and $u_{j-1}^{\prime}$ obey $(6)$, thus the field can be solved $[4,5]$, which gives the result using PE with ASE.

$$
u_{j}^{\prime}=\exp (A \Delta x) \cdot \exp (B \Delta x) u_{j-1}^{\prime}=F_{2}\left\langle u_{j-1}^{\prime}\right\rangle
$$

where $F_{2}=\exp (A \Delta x) \cdot \exp (B \Delta x)$.

In the following derivations, the Hadamard product is introduced to describe the product and division of elements in the vector, where they are expressed as "o" and "./," respectively.

The ASE $\mathfrak{R}_{j}$ at the $j$ th range step is shown as

$$
\mathfrak{R}_{j}=\left|u_{j}-u_{j}^{\prime}\right|=\left|e^{(B+A) \Delta x} u_{j-1}-e^{A \Delta x} e^{B \Delta x} u_{j-1}^{\prime}\right|,
$$

where $u_{j}^{\prime}$ and $u_{j}$ contain $N_{z}$ vertical grid points, respectively.

For the convenience of derivation, we define the correction factor

$S_{j}=\left(\mathrm{F} 1\left\langle u_{j}^{\prime}\right\rangle-\mathrm{F} 2\left\langle u_{j}^{\prime}\right\rangle\right) \cdot / u_{j}^{\prime}=\left[e^{(B+A) \Delta x} u_{j}^{\prime}-e^{A \Delta x} e^{B \Delta x} u_{j}^{\prime}\right] \cdot / u_{j}^{\prime}$.

With the detailed derivation found in the Appendix, $S_{j}$ is rewritten as

$$
\begin{aligned}
S_{j}=-\frac{(\Delta x)^{2}}{4}\{ & {\left[\left(\frac{\partial n_{j}}{\partial z}\right)^{\circ 2}+n_{j} \circ \frac{\partial^{2} n_{j}}{\partial z^{2}}\right] } \\
& +2 i k\left(\begin{array}{c}
\left.\left.n_{j} \circ \frac{\partial n_{j}}{\partial z}+\frac{1}{a}\left[\begin{array}{c}
1 \\
1 \\
\vdots \\
1
\end{array}\right]\right) \sin \alpha\right\}
\end{array}\right\} .
\end{aligned}
$$

Referring to the iteration process of SSFT, we derive the ASE.

Given the initial field $u_{0}$, the field of next range step $x=\Delta x$ is shown as

$$
u_{1}=F_{1}\left\langle u_{0}\right\rangle
$$

$$
u_{1}^{\prime}=F_{2}\left\langle u_{0}\right\rangle .
$$

Using (12), the field $u_{1}$ is given by

$$
u_{1}=\left(F_{1}\left\langle u_{0}\right\rangle-F_{2}\left\langle u_{0}\right\rangle\right) \cdot / u_{0} \circ u_{0}+F_{2}\left\langle u_{0}\right\rangle=u_{j}^{\prime}+S_{0} \circ u_{0} .
$$

For the range step $x=2 \Delta x, u_{2}$ satisfies the following relationship

$$
\begin{aligned}
u_{2}-u_{2}^{\prime} & =\left(F_{1}\left\langle u_{1}^{\prime}\right\rangle-F_{2}\left\langle u_{1}^{\prime}\right\rangle\right) \cdot / u_{1}^{\prime} \circ u_{1}^{\prime}+F_{1}\left\langle S_{0} \circ u_{0}\right\rangle \\
& =\left(S_{1}+S_{0}\right) \circ u_{1}^{\prime}+S_{0}^{\circ 2} \circ u_{0} .
\end{aligned}
$$

Then we get

$$
u_{2}=u_{2}^{\prime}+\left(S_{1}+S_{0}\right) \circ u_{1}^{\prime}+S_{0}^{\circ 2} \circ u_{0} .
$$

And so on, the field of range $x=j \Delta x$ is provided by

$$
u_{j}=\sum_{k=0}^{j}\left\{\left[\sum_{1}^{C_{j}^{k}}\left(\prod_{p=0}^{k} S_{p}^{\circ t_{p}}\right)\right] \circ u_{k}^{\prime}\right\} \text {, }
$$

where $\sum_{p=0}^{k} t_{p}=(j-k), 0 \leq t_{p} \leq(j-k), t_{p}$ is the integer, and

$C_{j}^{k}$ represents the combination, which can be written as $C_{j}^{k}=k ! /[j !(k-j) !]$ using the factorials.

We now get

$$
\mathfrak{R}_{j}=\left|u_{j}-u_{j}^{\prime}\right|=\left|\sum_{k=0}^{j-1}\left\{\left[\sum_{1}^{C_{j}^{k}}\left(\prod_{p=0}^{k} S_{p}^{\circ t_{p}}\right)\right] \circ u_{k}^{\prime}\right\}\right| .
$$

For horizontally homogeneous atmosphere, the refractivity index $n$ only varies with $z$. Then the term $S_{j}$ in (13) is the same at each range and unified as $S_{0}$. Considering these relationships given in (14)-(19), $\mathfrak{R}_{j}$ is obtained in homogeneous medium

$$
\mathfrak{R}_{j}=\left|u_{j}-u_{j}^{\prime}\right|=\left|\sum_{i=1}^{j} C_{j}^{i} S_{0}^{\circ i} \circ u_{j-1}^{\prime}\right| .
$$

We can conclude from above derivation: with the $j$ increasing, the calculation complexity of $u_{j}$ increases; the ASE $\Re_{j}$ is affected by environment parameters $\left(n_{j}, \partial n_{j} / \partial z\right.$, and $\partial^{2} n_{j} / \partial z^{2}$ ), antenna parameters (the frequency $f$ ), calculation range step $\Delta x$, propagation elevation angle $\alpha$, and $u_{j}^{\prime}$; the derivation process is involved in higher order accumulation, multiplication, and power operation. In order to effectively reduce the ASE, we study its sensitivity by varying these parameters.

\section{Sensitivity Analysis}

On account of the derivation results of ASE, its sensitivity involved by various parameters is addressed using computer 
calculation and statistical analysis methods. Standard and nonstandard atmosphere are examined, where the nonstandard atmosphere takes evaporation duct model as an example.

Since the transmission loss is the most commonly used parameter to describe the radio propagation characteristics, we utilize this quantity instead of the field to discuss the sensitivity of ASE. The transmission loss calculated from $u_{j}^{\prime}$ and $u_{j}$ is expressed as $L_{\text {depart }}$ and $L_{\text {join }}$, respectively. Simulations are given to compare the differences between $L_{\text {depart }}$ and $L_{\text {join }}$.

Antenna parameters are shown in Table 1. The distributions of $L_{\text {depart }}$ and $L_{\text {join }}$ in a standard atmosphere are described in Figure 2 with the condition of $f=10 \mathrm{GHz}$, and $\Delta x=500 \mathrm{~m}$ (calculation domain: $60 \mathrm{~km} \times 75 \mathrm{~m}$ ). Besides, the distributions of $L_{\text {depart }}$ and $L_{\text {join }}$ in an evaporation duct are described in Figure 3 with the same calculation condition with Figure 2, where the simplified Paulus-Jeske (PJ) evaporation duct model [16] is taken with duct height of $20 \mathrm{~m}$.

Comparing Figure 2(a) to Figure 2(b), the effect of ASE on transmission loss is more prominent at the range greater than $20 \mathrm{~km}$. It is because that the cumulative effect of split error increases with the range increasing in a standard atmosphere. Comparing Figure 3(a) to Figure 3(b), the difference of transmission loss is greater inside the duct, especially nearby the ocean. The reason is that the greater gradient varieties of refractivity index along the height direction in (13) lead to greater ASE.

In order to further discuss the effect of environmental parameters, $\Delta x$, and $f$ on the ASE, two statistical methods are utilized: one dissimilarity method and one similarity method.

The first method defines the mean value $\Delta L$ of relative percentage difference which is calculated from the absolute difference between $L_{\text {join }}(x, z)$ and $L_{\text {depart }}(x, z)$, and dividing by $L_{\text {depart }}(x, z)$. Therefore, the mean characteristics of transmission loss difference in the given propagation domain are described.

$\Delta L=\frac{\sum_{x} \sum_{z}\left|\left(L_{\text {join }}(x, z)-L_{\text {depart }}(x, z)\right) / L_{\text {depart }}(x, z)\right| \times 100 \%}{N_{x} \cdot N_{z}}$.

The second method applies correlation coefficient $R$ between $L_{\text {depart }}$ and $L_{\text {join }}$; thus, the bias from the mean value is considered.

$$
R=\frac{\sum_{x} \sum_{z}\left[\left(L_{\text {join }}(x, z)-\mu_{\text {join }}\right)\left(L_{\text {depart }}(x, z)-\mu_{\text {depart }}\right)\right]}{\sqrt{\sum_{x} \sum_{z}\left[L_{\text {join }}(x, z)-\mu_{\text {join }}\right]^{2} \sum_{x} \sum_{z}\left[L_{\text {depart }}(x, z)-\mu_{\text {depart }}\right]^{2}}},
$$

where $\mu_{\text {join }}$ and $\mu_{\text {depart }}$ are the mean of $L_{\text {depart }}$ and $L_{\text {join }}$, respectively.
TABLE 1: Antenna parameters.

\begin{tabular}{lc}
\hline Parameters & Values \\
\hline Frequency & $1 \sim 12 \mathrm{GHz}$ \\
Antenna height & $10 \mathrm{~m}$ \\
Elevation angle & $0^{\circ}$ \\
Beam width & $0.5^{\circ}$ \\
Polarization & Horizontal polarization \\
\hline
\end{tabular}

With the two methods, the simulation is conducted with the frequencies of $\mathrm{L}$ band, $\mathrm{S}$ band, $\mathrm{C}$ band, and $\mathrm{X}$ band, which are commonly used for microwave electromagnetic propagation. In addition, the range steps are taken from $50 \mathrm{~m}$ to $500 \mathrm{~m}$.

3.1. Sensitivity Analysis in a Standard Atmosphere. We utilize the above-mentioned two methods to analyze the sensitivity in a standard atmosphere.

Figure 4 presents the distributions of $\Delta L$ and $R$ varying with $f$ and $\Delta x$ in a standard atmosphere. In the four frequency bands, $\Delta L$ increases with range step, but $R$ decreases. As noticed, the range step has weak effect on the frequency of L band, making $\Delta L$ smaller than $1 \%$ and $R$ greater than 0.99 . Thus, the ASE can be neglected for PE in this band. In order to control $\Delta L \leq 1 \%$ and $R>0.99$, the range step of smaller than $350 \mathrm{~m}$ should be taken for the frequency of $\mathrm{S}$ band. We should use the range step smaller than $150 \mathrm{~m}$ for the frequency of $\mathrm{C}$ band and smaller than $100 \mathrm{~m}$ for the frequency of $\mathrm{X}$ band.

3.2. Sensitivity Analysis in an Evaporation Duct. Two statistical methods are applied for analyzing the sensitivity in an evaporation duct.

Figure 5 provides the distributions of $\Delta L$ and $R$ varying with $f$ and $\Delta x$ in an evaporation duct, which is obviously different from Figure 4. Comparing Figure 5 to Figure 4, the difference between the maximum and the minimum of $\Delta L$ in Figure 5(a) is smaller than that in Figure 4(a), but the difference range of $R$ in Figure 5(b) is greater than that in Figure 4(b). Therefore, the effect of ASE on transmission loss in an evaporation duct is relatively greater to that in a standard atmosphere. In order to control $\Delta L \leq 1 \%$ and $R>0.99$, we utilize range step smaller than $300 \mathrm{~m}$ for the frequency of L band, smaller than $200 \mathrm{~m}$ for the frequency of $\mathrm{S}$ band, smaller than $150 \mathrm{~m}$ for the frequency of $\mathrm{C}$ band, and smaller than $150 \mathrm{~m}$ for the frequency of $\mathrm{X}$ band.

In contrast, examining the distribution of the overall metrics $(\Delta L$ and $R$ ) for the transmission loss inside the duct is shown in Figure 6. The comparisons of Figure 6, Figure 5, and Figure 4 show that $L_{\text {depart }}$ in agreement with $L_{\text {join }}$ is the least close inside evaporation duct, which leads to the greater difference between the maximum and the minimum of $R$ than that in an entire domain. This is because the first derivative of refractivity index dramatically varieties with heights and the second derivative is nonzero. Therefore, the ASE cannot be decreased by simply taking the smaller range step in the high frequency, such as $\mathrm{X}$ band. 


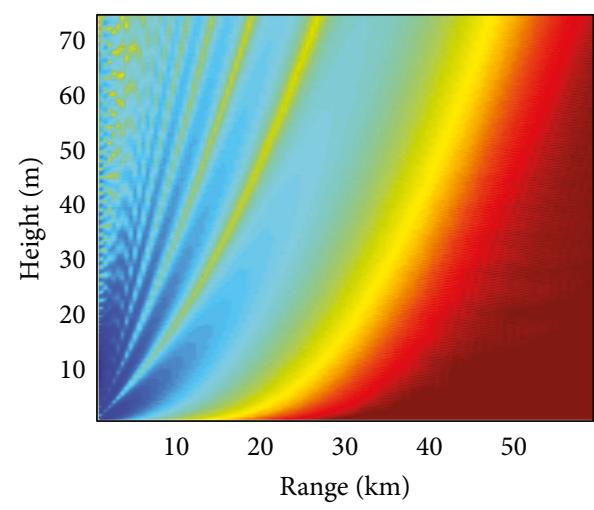

(a)

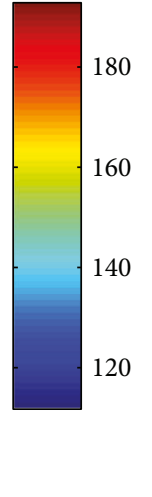

FIgURE 2: The transmission loss distribution in a standard atmosphere. (a) The distribution of $L_{\text {depart }}$.

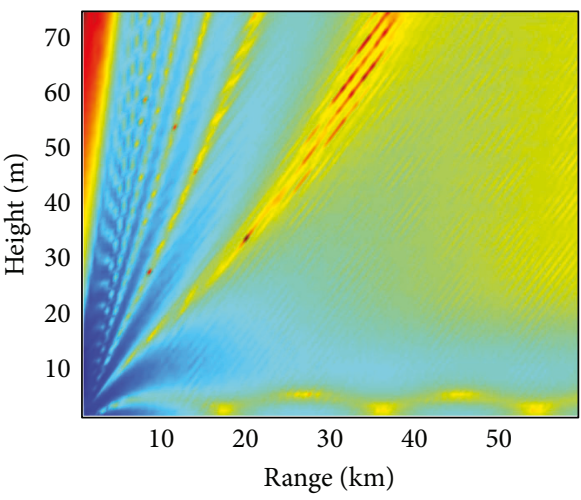

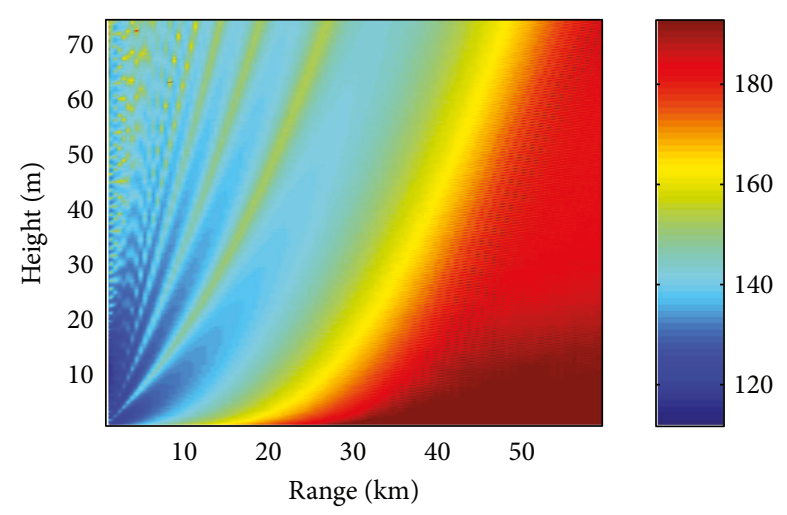

(b)

(b) The distribution of $L_{\text {join }}$.

FIgURE 3: The transmission loss distribution for a $20 \mathrm{~m}$ evaporation duct. (a) The distribution of $L_{\text {depart }}$ (b) The distribution of $L_{\text {join }}$.

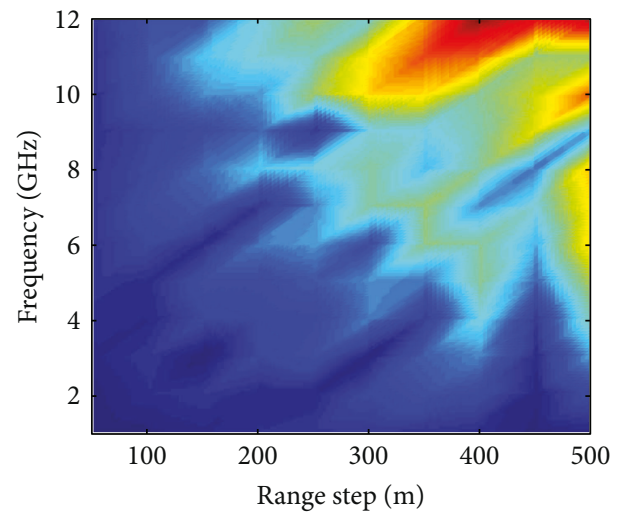

(a) (a)
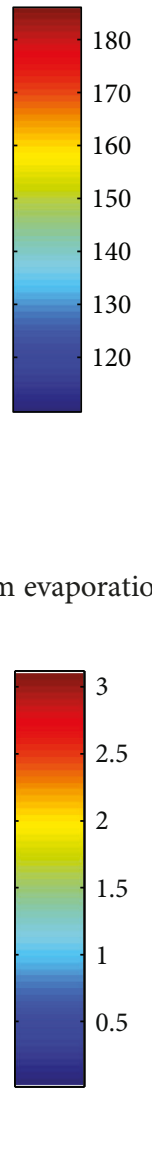

Figure 4: The distribution of $\Delta L$ and $R$ in a standard atmosphere. (a) $\Delta L$ and (b) $R$.

\section{Accumulated Split Error Correction Method}

On account of the above-mentioned simulations and analysis, the PE-accumulated split error correction method is proposed. This method is verified by transhorizon tropospheric duct test data.

We propose the correction method of ASE contained in $u_{j}^{\prime}$ on the basis of its definition and derivation in
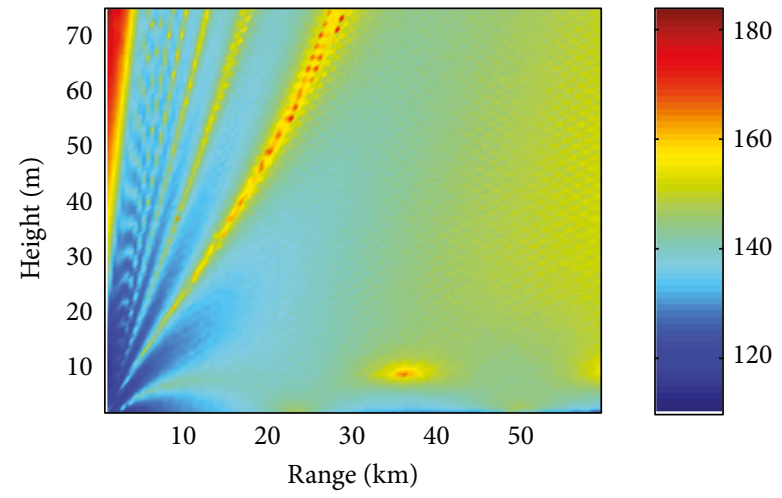

(b)

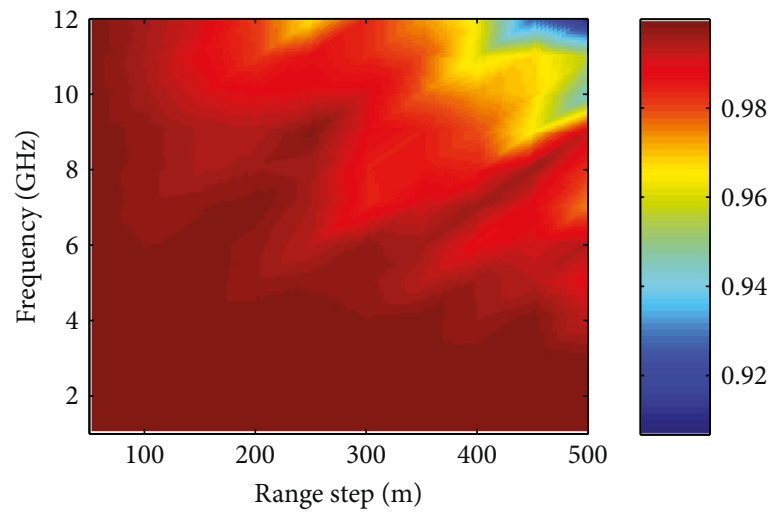

(b) 


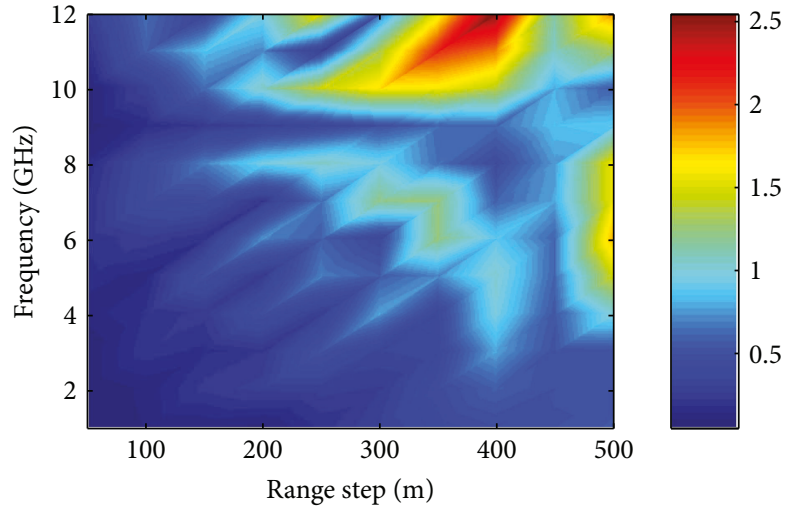

(a)

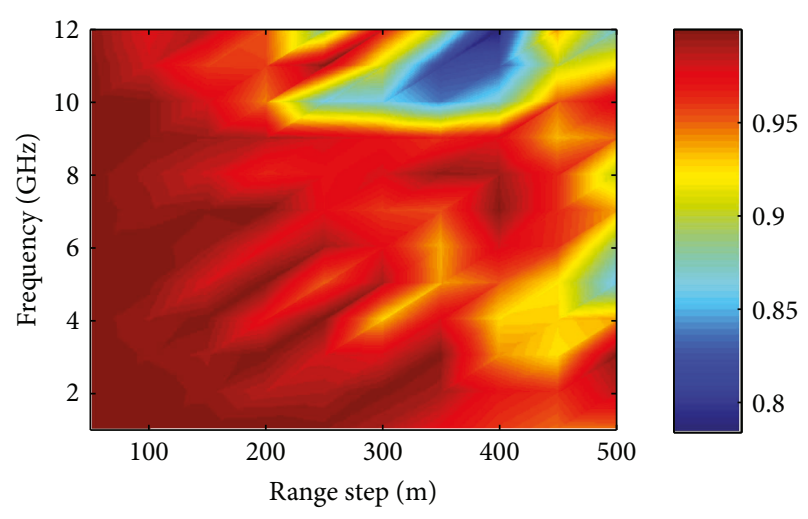

(b)

Figure 5: The distribution of $\Delta L$ and $R$ for the 20 m evaporation duct. (a) $\Delta L$ and (b) $R$.

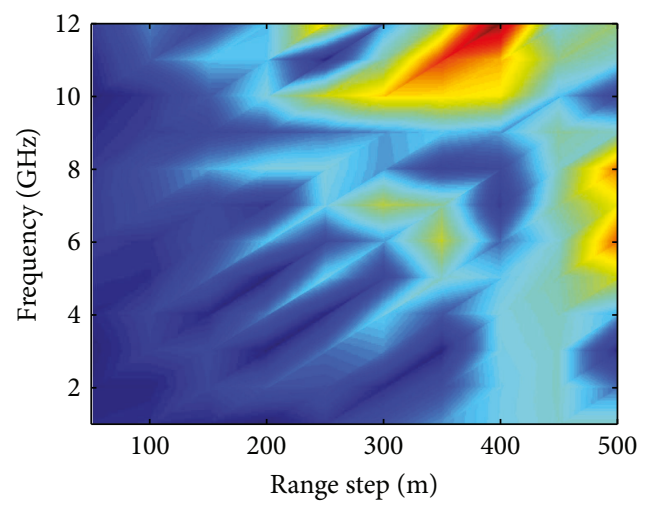

(a)
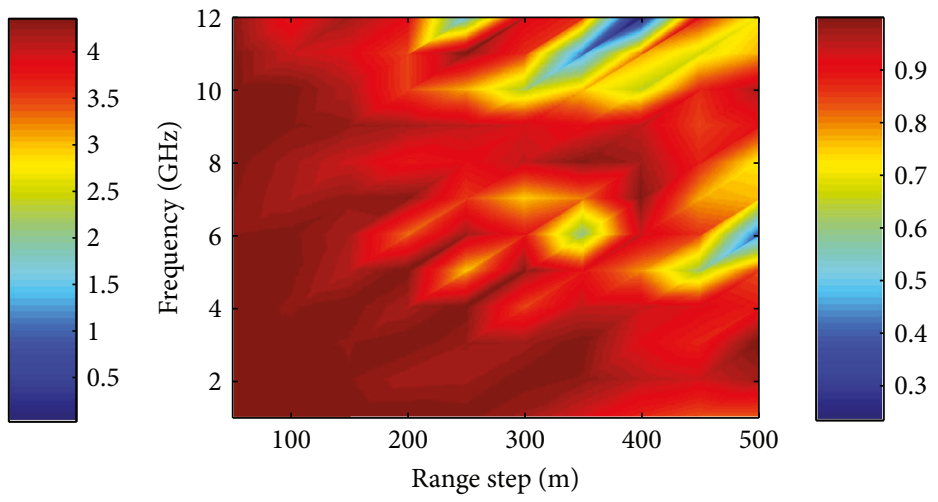

(b)

Figure 6: The distribution of $\Delta L$ and $R$ for the region of inside duct $(z<20 \mathrm{~m})$. (a) $\Delta L$ and (b) $R$.

(1) Input the antenna parameters.

(2) Build propagation environment using refractivity profile.

(3) Calculate the propagation field $u_{j}^{\prime}$ using PE [1].

On the conditions of (1) and (2), $u_{j}^{\prime}\left(j \in\left[0, N_{x}\right]\right)$ is computed using PE [1].

(4) Compute the ASE correction factor.

Using parameters from (1) and a given range step $\Delta x$, ASE correction factor $S_{j}\left(j \in\left[1, N_{x}\right]\right)$ is calculated by (13) in the environment of (2).

(5) Correct the ASE.

On the basis of (3) and (4), the ASE is corrected using (14)-(19).

(6) Output the field without the ASE.

Using (5) yields the field $u_{j}\left(j \in\left[1, N_{x}\right]\right)$.

As noticed in Figure 7, the SSFT to solve PE is involved in complex techniques, including the absorbing boundary, surface boundary modeling, and split-step calculation. Besides, the interaction process from (14) to (19) shows that higher order accumulation, multiplication, and power operation are introduced. Therefore, this correction method increases the calculation complexity to improve the accuracy of PE. Taking the standard atmosphere for an example, we provide the complexity and efficiency analysis. The simulation results are shown in Figure 8. These results are based on the two aspects as follows:

(1) The statistical relationship between calculation step range and execution time is analyzed for $\mathrm{PE}$ with ASE method and PE without ASE method, respectively. These results are simulated using the computer CPU of i7-4270HQ and shown in Figure 8(a).

(2) Given the upper threshold of error $\Delta L$, the statistical relationship between frequency and execution time is analyzed and given in Figure 8(b).

As noticed in Figure 8(a), the execution time decreases with the range step increasing for the two methods. However, the execution time of PE without ASE method (red color) is much greater than that of PE with ASE method (blue color) on the condition of the same range step, especially for smaller 
(1) Input antenna parameters

Calculate field using PE

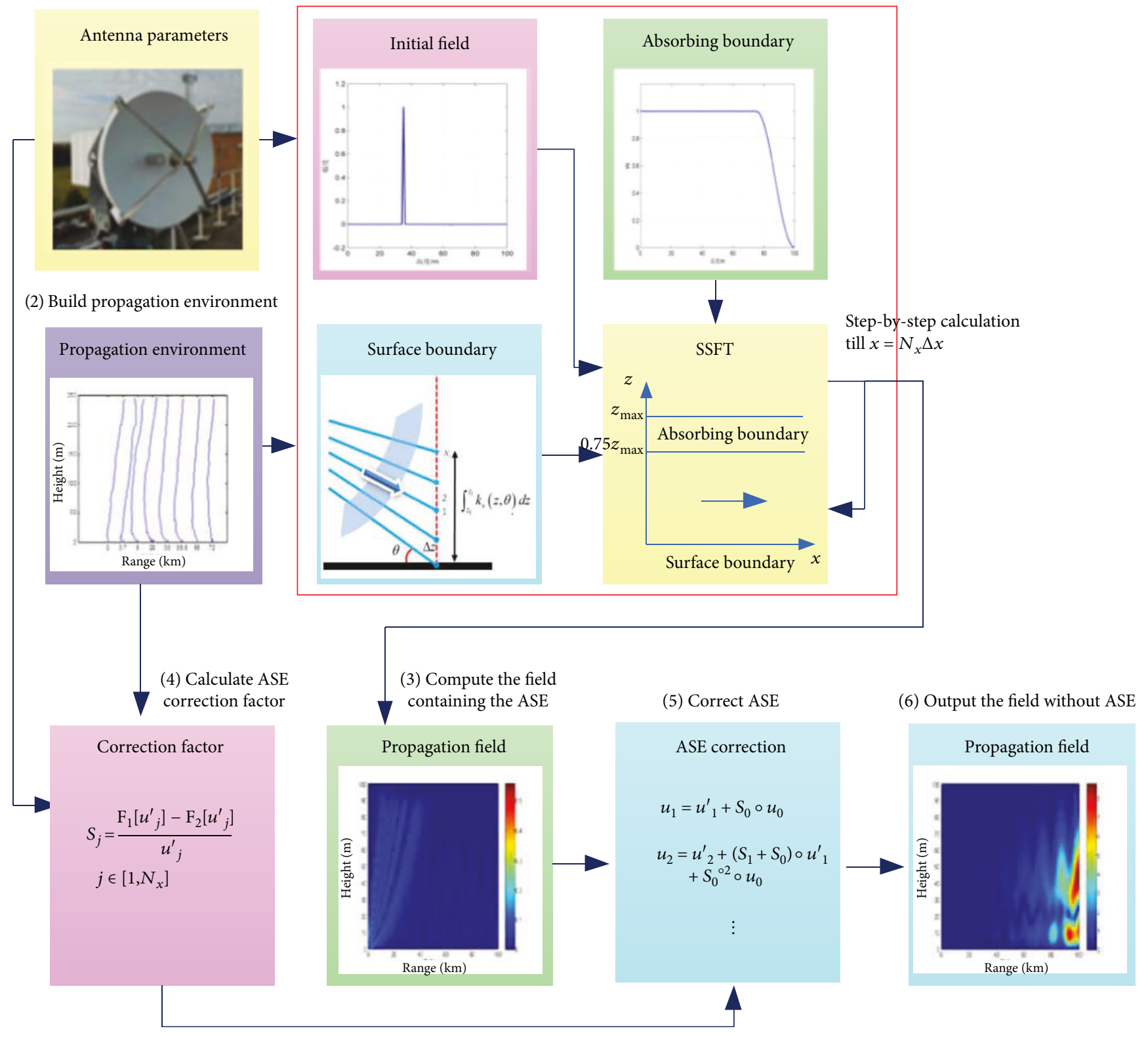

FIgure 7: The ASE correction diagram.

range step. This result agrees with foregoing analysis that the calculation complexity increases by involving in complex correction factor. On the basis of accepted error threshold and execution time in Figure 8(b), the range step can be determined for a given frequency by referring to Figure 8(a). Therefore, Figure 8 (b) gives a reference for realistic application.

4.2. Experimental Example. The main purpose of this section is to verify the performance of the ASE correction method of PE.

The sea clutter data (Figure 9(a)) and corresponding data of a refractivity profile (Figure 9(b)) measured in Huanghai, China, August 1, 2014 are considered here. As noticed in Figure 9(a), the north direction is the azimuth $0^{\circ}$, and the sea clutter at $58.5^{\circ}$ azimuth is used as an example to analyze the performance of parabolic equation ASE correction method.

The radar antenna parameters are given in Table 2.

Since there is a clear weather for this measured data, smooth sea surface and range-independent refractivity profile are considered. By filtering the weather echo, ground echo, noise, and so on of sea clutter strength $X$ in Figure $9(\mathrm{a})$, we can obtain the sea clutter power $P_{\text {obs }}$ at range $r$ using

$$
P_{\text {obs }}=0.5(X-2)-32-20 \log (r)-C-30 \text {, }
$$

where $C$ takes into account radar parameters and so on. 


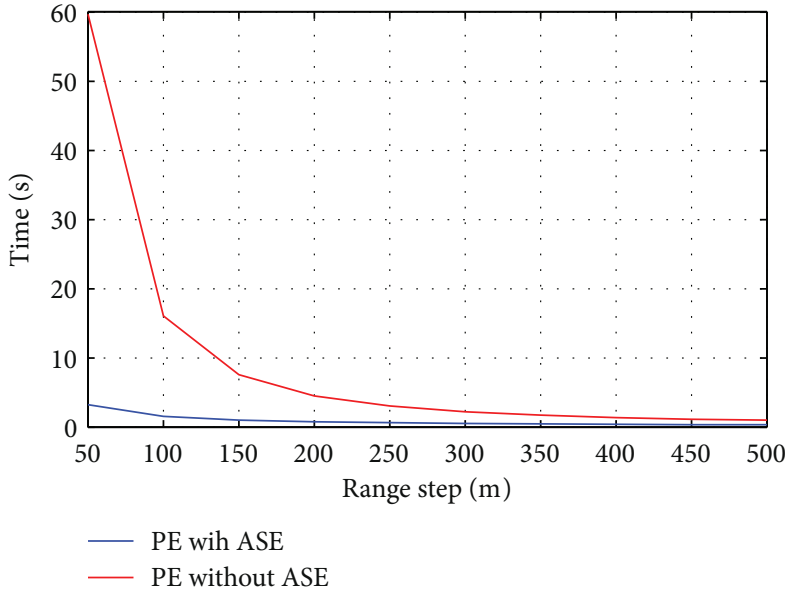

(a)

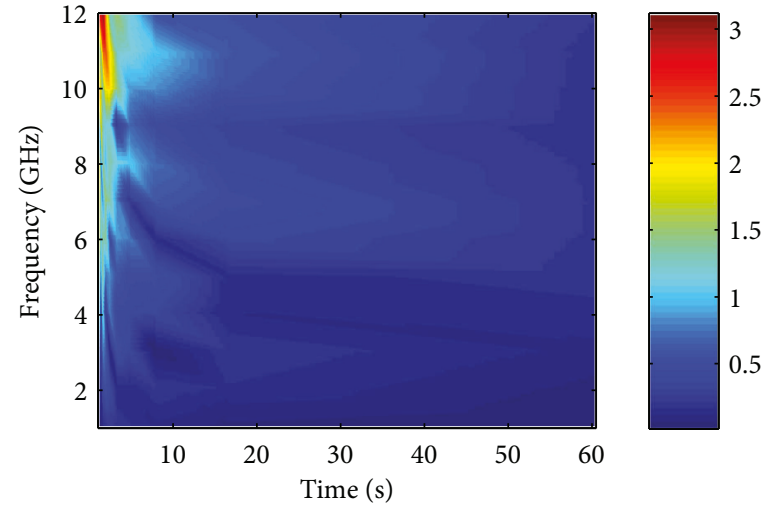

(b)

Figure 8: The complexity and efficiency analysis of PE. (a) The statistical relationship between calculation step range and execution time. (b) The statistical relationship between frequency and execution time.

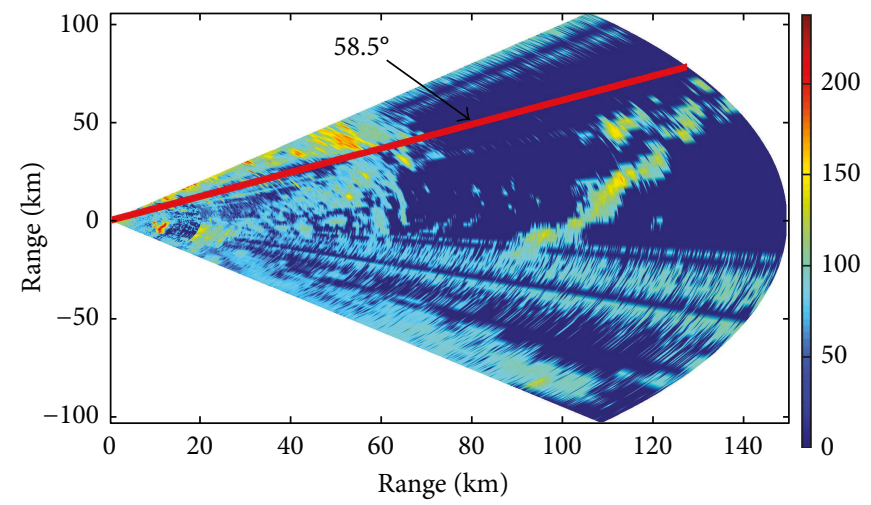

(a)

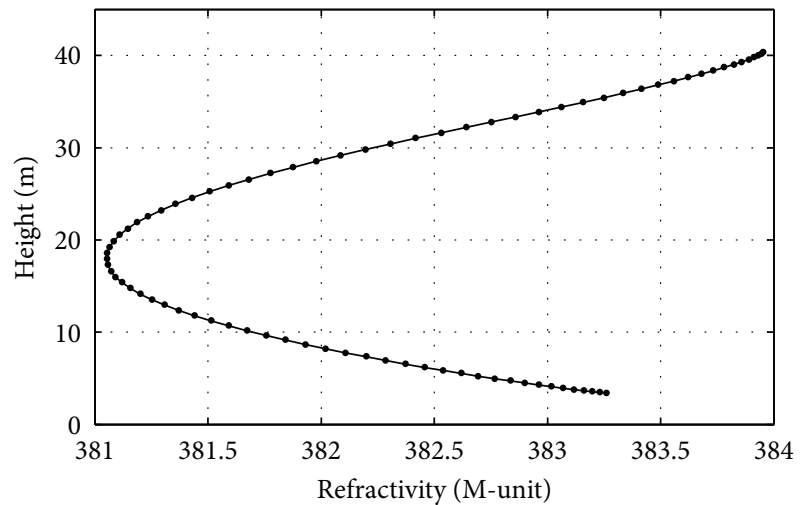

(b)

FIgURE 9: Measured data distribution. (a) Radar sea clutter strength. (b) Refractivity profile.

TABLE 2: Antenna parameters.

\begin{tabular}{lc}
\hline Parameters & Values \\
\hline Frequency & $9.6 \mathrm{GHz}$ \\
Antenna height & $35 \mathrm{~m}$ \\
Elevation angle & $0^{\circ}$ \\
Beam width & $1.5^{\circ}$ \\
Polarization & Vertical polarization \\
\hline
\end{tabular}

Applying the measured modified refractivity $M$ into the radar equation [17], the power can be computed by

$$
P_{c}(M, r)=-2 L(M, r)+10 \lg r+\sigma^{0}+C,
$$

where $L(M, r)$ is the transmission loss at range $r$ calculated by PE with ASE and without ASE method; $\sigma^{0}$ is the radar cross section of the sea surface.

In order to decrease the effect of range step on calculation results, we take $\Delta x=150 \mathrm{~m}$. The field $u_{j}^{\prime}$ is computed as the first step result. Then the field $u_{j}$ can be obtained. On the basis of the field, the theoretical power with $\operatorname{ASE} P_{c}^{\prime}(M, r)$ and power without $\operatorname{ASE} P_{c}(M, r)$ is shown in Figure 10, respectively, where the mean of error between $P_{c}^{\prime}$ and $P_{\text {obs }}$ is $12.3 \mathrm{~dB}$, but that between $P_{c}$ and $P_{\text {obs }}$ is $9.4 \mathrm{~dB}$. Therefore, the parabolic equation ASE correction method can effectively improve the calculation accuracy.

\section{Conclusion}

In this paper, we derived the ASE due to exponent split using SSFT numerical algorithm to solve PE. In addition, sensitivity analysis of ASE in standard and nonstandard atmosphere is addressed using two statistical methods. On account of above studies, we propose an ASE correction method for PE. We also verify that PE without ASE method has a greater accuracy by using the measured data from Huanghai, China. Although the ASE of SPE is only derived and simulated, the study idea we developed is also applied to WPE. This is also our future work. 


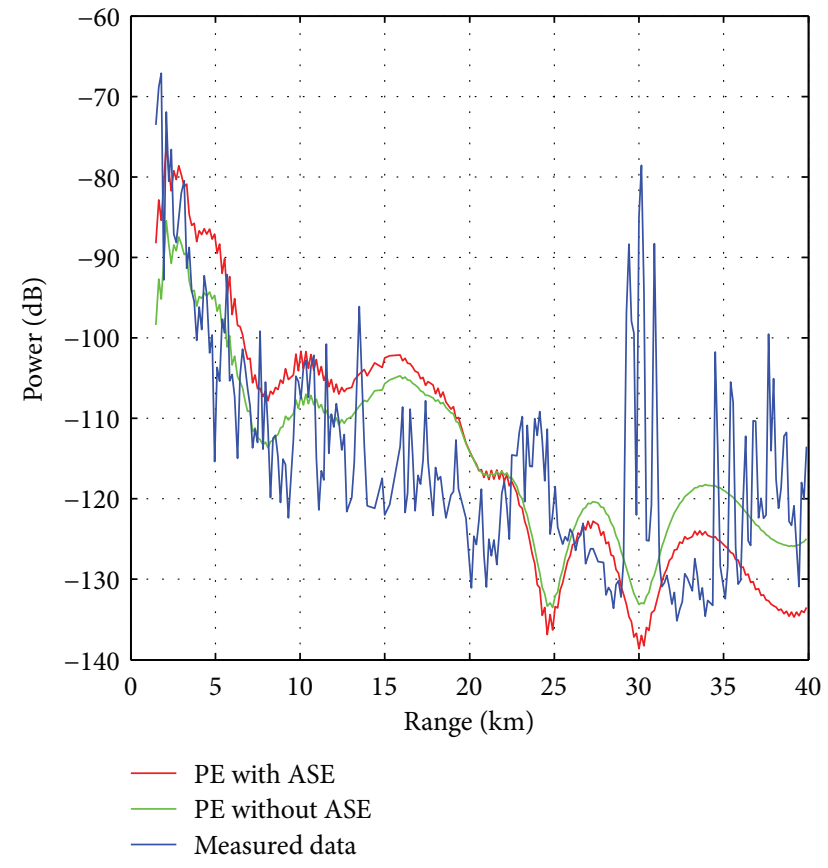

FIgURe 10: Sea clutter power distribution.

\section{Appendix}

In this section, we will derive the correction factor in (12).

Using the Taylor expansions, we express this formula with the second order in $\Delta x$

$$
S_{j} \approx \frac{1}{2}\left[\left(B A u_{j}^{\prime}-A B u_{j}^{\prime}\right) \cdot(\Delta x)^{2}\right] \cdot / u_{j}^{\prime} \cdot
$$

Substituting the two operator terms $A$ and $B$ into (A.1) yields.

$$
\begin{aligned}
S_{j}=-\frac{(\Delta x)^{2}}{4}\left\{\left[\left(\frac{\partial n_{j}}{\partial z}\right)^{\circ 2}+n_{j} \circ \frac{\partial^{2} n_{j}}{\partial z^{2}}\right] \circ u_{j}^{\prime}\right. \\
\\
\left.+2 \frac{\partial u_{j}^{\prime}}{\partial z} \circ\left(n_{j} \circ \frac{\partial n_{j}}{\partial z}+\frac{1}{a}\left[\begin{array}{c}
1 \\
1 \\
\vdots \\
1
\end{array}\right]\right)\right\} \cdot / u_{j}^{\prime},
\end{aligned}
$$

where $n_{j}$ is the refractivity index varied with height at $j$ th range step, and the vector dimension of $S_{j}$ is $N_{z}$. Besides, the vector $\left[\begin{array}{llll}1 & 1 & \ldots & 1\end{array}\right]^{T}$ has the same dimension with $n_{j}$.

We introduce the reduced function associated with the paraxial direction $x$

$$
u^{\prime}(x, z)=U^{\prime}(x, z) e^{-i k x}
$$

where $U^{\prime}(x, z)$ satisfies the two-dimensional scalar wave equation [4].

We consider a plane wave of the form

$$
U^{\prime}(x, z) \approx e^{i k(x \cos \alpha+z \sin \alpha)}
$$

of the amplitude 1 and propagating at elevation angle $\alpha$ and then substituting (A.4) into (A.3) obtains

$$
u^{\prime}(x, z)=e^{i k[x(\cos \alpha-1)+z \sin \alpha]} .
$$

Using this formula, we have

$$
\frac{\partial u^{\prime}}{\partial z}=e^{-i k x} e^{i k x \cos \alpha} \cdot i k \sin \alpha \cdot e^{i k z \sin \alpha}=i k \sin \alpha \cdot u^{\prime} \text {. }
$$

With the substitution of (A.6) into (A.2), we see

$$
\begin{aligned}
S_{j}=-\frac{(\Delta x)^{2}}{4}\{ & {\left[\left(\frac{\partial n_{j}}{\partial z}\right)^{\circ 2}+n_{j} \circ \frac{\partial^{2} n_{j}}{\partial z^{2}}\right] } \\
& \left.+2 i k\left(n_{j} \circ \frac{\partial n_{j}}{\partial z}+\frac{1}{a}\left[\begin{array}{c}
1 \\
1 \\
\vdots \\
1
\end{array}\right]\right) \sin \alpha\right\}
\end{aligned}
$$

\section{Conflicts of Interest}

The authors declare that they have no conflicts of interest.

\section{References}

[1] P. Zhang, L. Bai, Z. Wu, and L. Guo, "Applying the parabolic equation to tropospheric groundwave propagation: a review of recent achievements and significant milestones," IEEE Antennas and Propagation Magazine, vol. 58, no. 3, pp. 3144, 2016.

[2] L. M. Brekhovskikh, Waves in Layered Media, Academic Press, San Diego, California, 2nd edition, 1980.

[3] G. B. Baumgartner, H. V. Hitney, and R. A. Pappert, "Duct propagation modelling for the integrated-refractive-effects prediction system (IREPS)," IEE Proceedings F - Communications, Radar and Signal Processing, vol. 130, no. 7, pp. 630642, 1983.

[4] M. F. Levy, Parabolic Equation Methods for Electromagnetic Wave Propagation, The Institution of Electrical Engineers Press, London, 2000.

[5] J. R. Kuttler and G. D. Dockery, "Theoretical description of the parabolic approximation/Fourier split-step method of representing electromagnetic propagation in the troposphere," Radio Science, vol. 26, no. 2, pp. 381-393, 1991.

[6] C. Yardim, P. Gerstoft, and W. S. Hodgkiss, "Sensitivity analysis and performance estimation of refractivity from clutter techniques," Radio Science, vol. 44, no. 1, pp. 1-16, 2009. 
[7] D. Dockery and J. R. Kuttler, "An improved impedanceboundary algorithm for Fourier split-step solutions of the parabolic wave equation," IEEE Transactions on Antennas and Propagation, vol. 44, no. 12, pp. 1592-1599, 1996.

[8] L. Junwang, W. Hong-Guang, W. Zhen-Sen, and L. Lei, "PSO algorithm of retrieving surface ducts by Doppler weather radar echoes," Progress In Electromagnetics Research B, vol. 65, no. 1, pp. 19-33, 2016.

[9] Z. S. Wu, L. Li, L. K. Lin, and Z. W. Zhao, "Study on the maximum calculation height and the maximum propagation angle of the troposcatter wide-angle parabolic equation method," IET Microwaves, Antennas \& Propagation, vol. 10, no. 6, pp. 686-691, 2016.

[10] F. Vasileios and E. Christopher, "Duct heights inferred from radar sea clutter using proper orthogonal bases," Radio Science, vol. 51, no. 10, pp. 1614-1626, 2016.

[11] A. A. Zaporozhets and M. F. Levy, "Modelling of radiowave propagation in urban environment with parabolic equation method," Electronics Letters, vol. 32, no. 17, p. 1615, 1996.

[12] K. Arshad, F. Katsriku, and A. Lasebae, "An investigation of wave propagation over irregular terrain and urban streets using finite elements," in Proceedings of the 6th WSEAS International Conference on TELECOMMUNICATIONS and INFORMATICS, pp. 105-110, Dallas, TX, USA, March 2007.

[13] F. Tappert, Wave Propagation and Underwater Acoustics, Springer, Berlin, Heidelberg, 1977.

[14] G. D. Dockery, "Modeling electromagnetic wave propagation in the troposphere using the parabolic equation," IEEE Transactions on Antennas and Propagation, vol. 36, no. 10, pp. 1464-1470, 1988.

[15] H. Hui-bin, M. Jun-jie, and C. Shun-lian, "Error analysis of wide-angle parabolic equation for radio propagation," Chinese Journal of Radio Science, vol. 21, no. 2, pp. 199-203, 2006.

[16] L. T. Rogers, C. P. Hattan, and J. K. Stapleton, "Estimating evaporation duct heights from radar sea echo," Radio Science, vol. 35, no. 4, pp. 955-966, 2000.

[17] P. Gerstoft, L. T. Rogers, and J. K. Krolik, "Inversion for refractivity parameters from radar sea clutter," Radio Science, vol. 38, no. 3, pp. 1-22, 2003. 


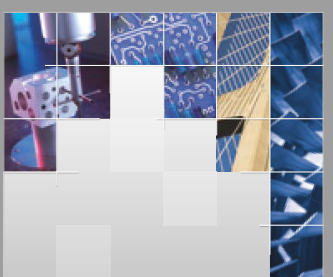

\section{Enfincering}
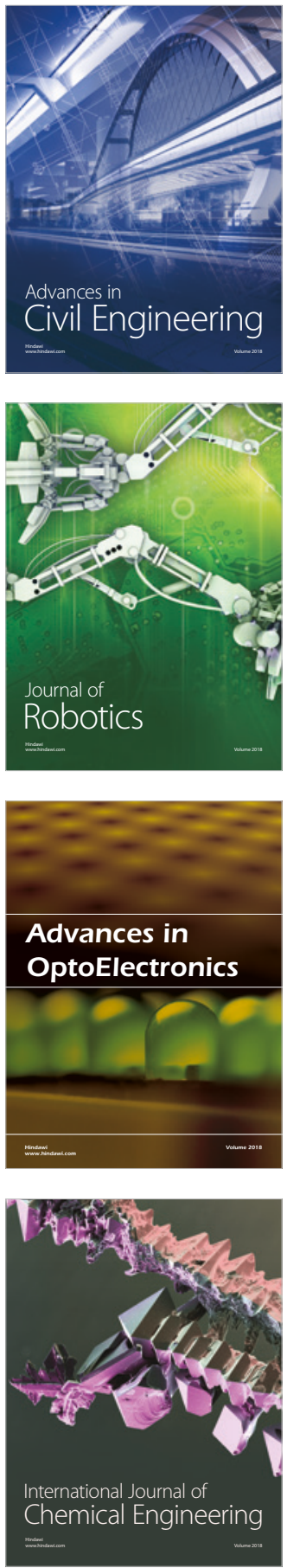

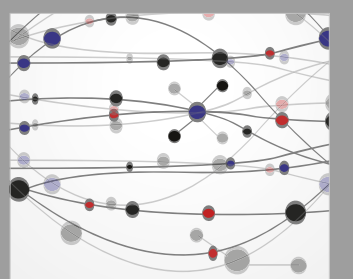

\section{Rotating \\ Machinery}

The Scientific World Journal

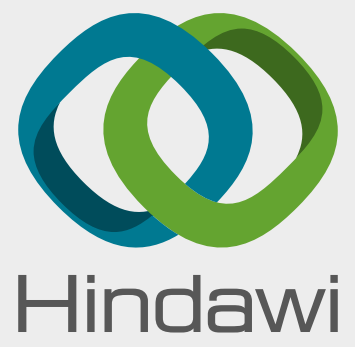

Submit your manuscripts at

www.hindawi.com
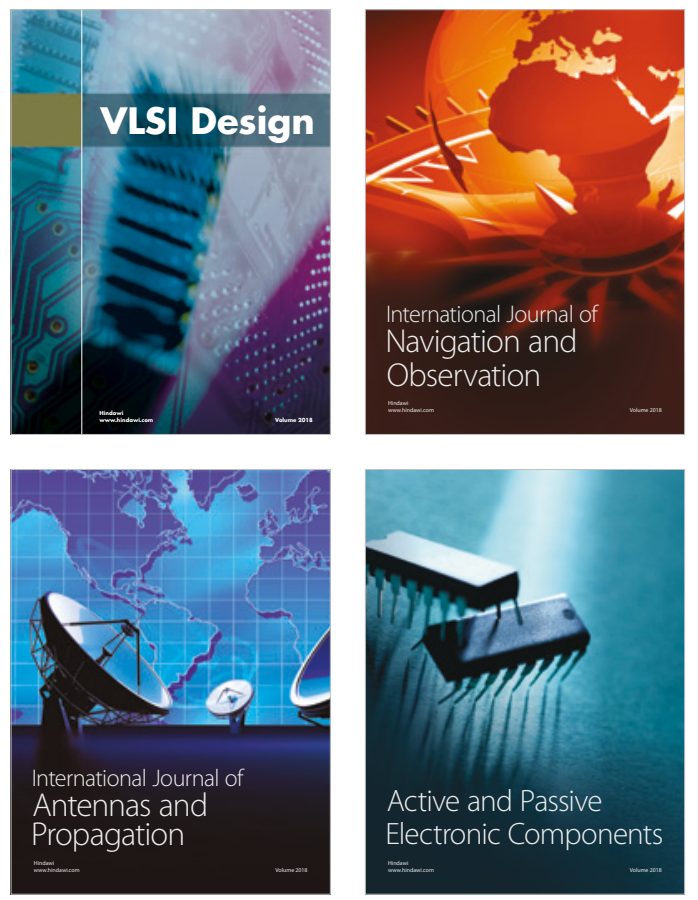
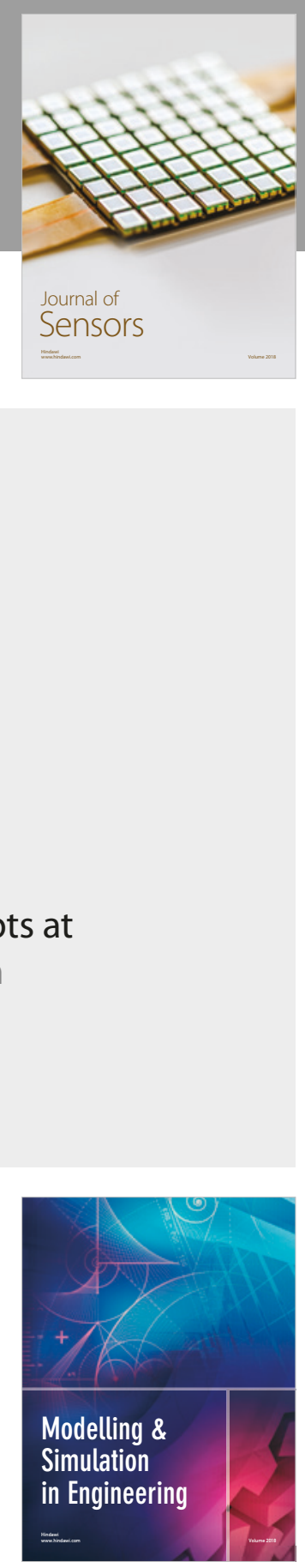

\section{Advances \\ Multimedia}
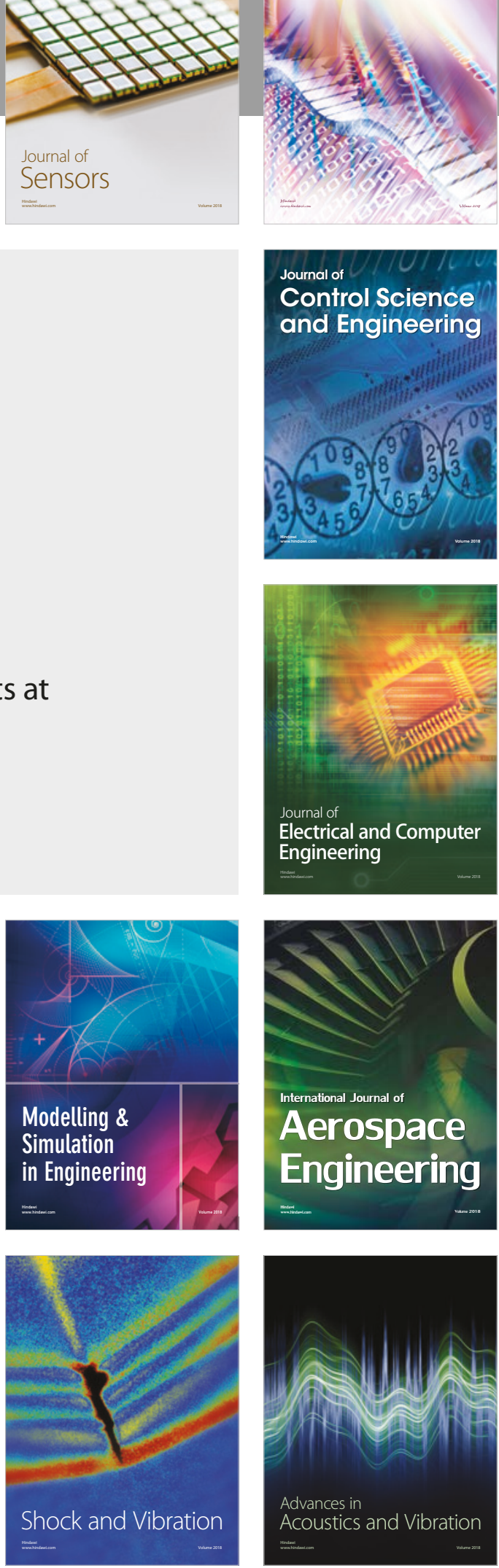